\title{
Experimental Results Validating the Near-Field to Far-Field Transforma- tion Technique with Helicoidal Scan
}

\author{
F. D’Agostino, F. Ferrara, C. Gennarelli*, R. Guerriero and M. Migliozzi
}

D.I.I.I.E - University of Salerno, via Ponte Don Melillo, 84084 Fisciano (Salerno), Italy

\begin{abstract}
This paper concerns the experimental validation of the near-field - far-field transformation technique with helicoidal scanning. Such a technique, based on the theoretical results concerning the nonredundant sampling representations of the electromagnetic fields, makes use of a two-dimensional optimal sampling interpolation formula to reconstruct the near-field data needed to perform the classical near-field - far-field transformation with cylindrical scan. The comparison of the far-field patterns reconstructed from the acquired helicoidal measurements with those obtained from the data directly measured on the classical cylindrical grid assesses the effectiveness of the near-field - far-field transformation using this innovative scanning technique. Its validity is further confirmed by the very good agreement with the direct farfield measurements.
\end{abstract}

Keywords: Helicoidal scanning, NF-FF transformation techniques, Nonredundant sampling representations.

\section{INTRODUCTION}

For electrically large antennas, it is impractical to directly measure the radiation patterns on a conventional far-field (FF) range. Therefore, for such antennas, it is useful to exploit near-field (NF) measurements to recover the FF patterns via NF-FF transformation techniques [1-3]. In addition, the NF measurements may be performed in a controlled environment, such as in an anechoic chamber, thus overcoming those drawbacks due to weather conditions, electromagnetic (EM) interference, etc., which cannot be eliminated in FF outdoor measurements. The reduction of the time required for the acquisition of the NF data is becoming a very important issue for the antenna community. In fact, this time is currently very much greater than that needed to carry out the corresponding NF-FF transformation. A significant reduction of the number of required NF data (and, as a consequence, the measurement time) has been obtained for all the conventional scannings [4-10] by applying the theoretical results on the nonredundant sampling representations of EM fields [11] and the optimal sampling interpolation (OSI) expansions [12]. The use of the modulated scattering technique employing arrays of scattering probes, which allows a very fast electronic scanning, has been also proposed in [13] to reduce the time needed for the acquisition of the NF data. However, antenna testing NF facilities based on such a technique are not very flexible. A more convenient way of reducing the measurement time is the use of innovative spiral scanning techniques. They have been implemented, as suggested by Rahmat-Samii et al. in [14], by means of continuous and synchronized movements of the positioning systems of the probe and antenna under test (AUT). In particular, NF-FF transformations using the helicoidal scanning [15, $16]$, the planar $[16,17]$ and spherical $[16,18]$ spiral scannings

*Address correspondence to this author at the Dipartimento di Ingegneria dell'Informzione ed Ingegneria Elettrica, (D.I.I.I.E.) - University of Salerno, via Ponte Don Melillo, 84084 Fisciano (Salerno), Italy; Tel: +39089964 297; Fax: +39 089964 218; E-mail: gennar@diiie.unisa.it have been developed. They are based on the aforementioned nonredundant sampling representations and OSI expansions. Accordingly, the NF data needed by the corresponding NFFF transformation can be reconstructed by interpolating the nonredundant ones acquired on the spiral. The required twodimensional algorithm has been obtained [16]: a) by assuming the AUT enclosed in the smallest sphere able to contain it; b) by developing a nonredundant sampling representation of the voltage on the spiral; c) by choosing the spiral step equal to the sample spacing required to interpolate the data along a meridian curve.

This paper deals with the experimental validation of the NF-FF transformation with the helicoidal scanning (Fig. 1). Such a validation has been carried out at the laboratory of antenna characterization of the University of Salerno, where an advanced cylindrical NF measurement facility supplied by MI Technologies is available. The validity of the technique is further confirmed by the very good agreement with the direct FF measurements performed in the same laboratory.

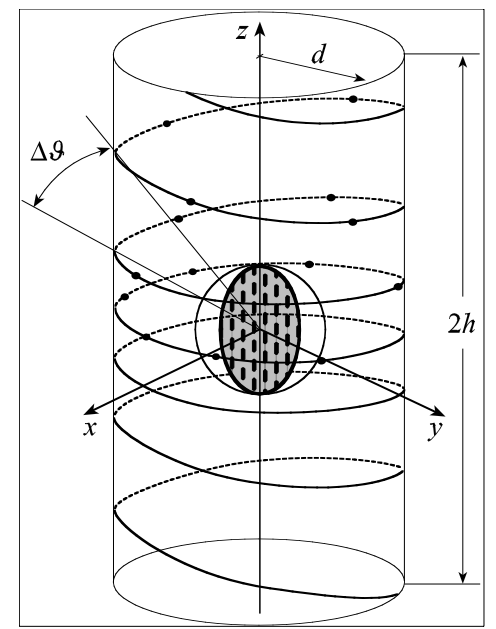

Fig. (1). Helicoidal scanning. 


\section{NONREDUNDANT REPRESENTATION OF THE PROBE VOLTAGE}

The main results concerning the reconstruction of the voltage from the knowledge of a nonredundant number of its samples acquired by the measurement probe along a helix [16] are summarized in the following.

Let us consider an AUT and a nondirective probe scanning a proper helix lying on a cylinder of radius $d$ (see Fig. 1) and adopt the spherical coordinate system $(r, \vartheta, \varphi)$ for denoting the observation point $P$. Since the voltage $V$ measured by such a probe has the same effective spatial bandwidth of the field [19], the theoretical results relevant to the nonredundant representation of EM fields [11] can be applied to such a voltage. Accordingly, if the AUT is enclosed in the smallest sphere of radius $a$ able to contain it and the helix is described by an analytical parameterization $\underline{r}=\underline{r}(\xi)$, it is possible to introduce the probe "reduced voltage"

$\tilde{V}(\xi)=V(\xi) \mathrm{e}^{\mathrm{j} \gamma(\xi)}$

where $\gamma(\xi)$ is a phase function to be determined. The error occurring when $\tilde{V}$ is approximated by a spatially bandlimited function becomes negligible as the band-width exceeds a critical value $W_{\xi}$ [11]. In fact, it exhibits a step-like behaviour whose transition occurs at $W_{\xi}$ [20]. Accordingly, such a bandlimitation error can be controlled [21] by choosing a bandwidth equal to $\chi^{\prime} W \xi$, where $\chi^{\prime}$ is an enlargement factor (slightly greater than unity for electrically large antennas).

The coordinates of a point $Q$ on the helix, when imposing the passage of the helix through a given point $P_{0}$ at $z=h$ (Fig. 1) of the generatrix at $\varphi=0$, are:

$$
\left\{\begin{array}{l}
x=d \cos \left(\phi-\phi_{i}\right) \\
y=d \sin \left(\phi-\phi_{i}\right) \\
z=d \cot \theta
\end{array}\right.
$$

where $\phi_{i}$ is the value at $P_{0}$ of the angular parameter $\phi$ describing the helix and $\theta=k \phi, k$ determining the helix step. Such a helix can be constructed as intersection of the cylindrical surface with the line from the origin to a point moving on a spiral which wraps the sphere enclosing the AUT. In order to allow the two-dimensional interpolation, the helix step $\Delta \theta$ must be equal to the sample spacing needed to interpolate the voltage along a generatrix $[11,16]$. Then, $k$ is such that the step, determined by the consecutive intersections $Q(\phi)$ and $Q(\phi+2 \pi)$ of the helix with the cylinder generatrix, is $\Delta \theta=\Delta \vartheta=2 \pi /(2 M+1)$, with $M=\operatorname{Int}\left[\chi M^{\prime}\right]+1$ and $M^{\prime}=$ $\operatorname{Int}\left[\chi^{\prime} \beta a\right]+1$. Accordingly, since $\Delta \theta=2 \pi k$, it follows that $k$ $=1 /(2 M+1)$. The function $\operatorname{Int}[x]$ gives the integer part of $x$, $\beta$ is the wavenumber, and $\chi>1$ is an oversampling factor.

According to the results in $[11,16]$, a nonredundant sampling representation of the voltage on the helix can be obtained by using the following expressions for the optimal phase function and parameterization:

$$
\begin{aligned}
& \gamma(s)=\frac{\beta}{2} \int_{0}^{s}\left[\max _{\underline{r}^{\prime}} \hat{R} \cdot \hat{t}+\min _{\underline{r}^{\prime}} \hat{R} \cdot \hat{t}\right] \mathrm{d} s \\
& \xi=\xi(s)=\frac{\beta}{2 W_{\xi}} \int_{0}^{s}\left[\max _{\underline{r}^{\prime}} \hat{R} \cdot \hat{t}-\min _{\underline{r^{\prime}}} \hat{R} \cdot \hat{t}\right] \mathrm{d} s
\end{aligned}
$$

where $s$ is the arclength of the helix, $\hat{t}$ is the unit vector tangent to it at $Q$, and $\hat{R}$ is the unit vector from the source point to $Q$. It can be verified that the extreme values of the inner product $\hat{R} \cdot \hat{t}$ are determined by considering the intersection of the plane defined by $\hat{t}$ and the unit vector $\hat{r}$ (pointing from the origin to $Q$ ) with the cone having vertex at $Q$ and the generatrices coincident with the tangents to the source ball. Denoting by $\hat{R}_{1,2}$ the related unit vectors and by $\varepsilon$ the angle between $\hat{r}$ and $\hat{t}$ (Fig. 2), it results [16]:

$$
\begin{aligned}
& \left(\hat{R}_{1}+\hat{R}_{2}\right) / 2=\hat{r} \sin \delta=\hat{r} \sqrt{1-a^{2} / r^{2}} \\
& \left(\hat{R}_{1}-\hat{R}_{2}\right) \cdot \hat{t} / 2=\cos \delta \sin \varepsilon=(a / r) \sin \varepsilon
\end{aligned}
$$

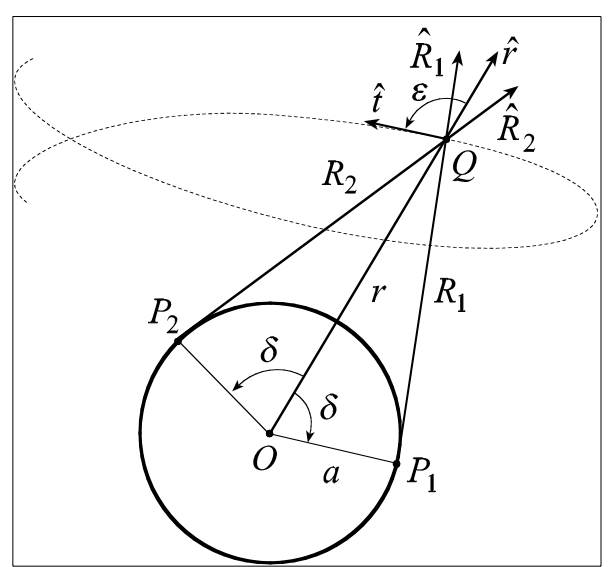

Fig. (2). Geometry of the problem in the plane $\hat{r}, \hat{t}$.

By substituting (5) into (3) and taking into account that $\mathrm{d} r=\hat{r} \cdot \hat{t} \mathrm{~d} s$, we obtain:

$\gamma=\beta \int_{0}^{r} \sqrt{1-\left(\frac{a}{r^{\prime}}\right)^{2}} \mathrm{~d} r^{\prime}=\beta \sqrt{r^{2}-a^{2}}-\beta a \cos ^{-1}\left(\frac{a}{r}\right)$

On the other hand

$\mathrm{d} s=\left(d / \sin ^{2} k \phi\right) \sqrt{k^{2}+\sin ^{4} k \phi} \mathrm{d} \phi$

and

$\sin \varepsilon=\sqrt{1-(\hat{r} \cdot \hat{t})^{2}}$

wherein

$\hat{r} \cdot \hat{t}=-k \cos k \phi / \sqrt{k^{2}+\sin ^{4} k \phi}$ 
By taking into account (9) and substituting relations (6) and (8) in (4), it results:

$\xi=\frac{\beta a}{W_{\xi}} \int_{0}^{\phi} \sqrt{k^{2}+\sin ^{2} k \phi^{\prime}} \mathrm{d} \phi^{\prime}$

As can be seen, the optimal parameter $\xi$ is proportional to the curvilinear abscissa along the spiral wrapping the sphere modelling the AUT. Since such a spiral is a closed curve, it is convenient to choose the bandwidth $W \xi$ such that $\xi$ covers a $2 \pi$ range when the whole curve on the sphere is described. As a consequence,

$W_{\xi}=\frac{\beta a}{\pi} \int_{0}^{(2 M+1) \pi} \sqrt{k^{2}+\sin ^{2} k \phi^{\prime}} \mathrm{d} \phi^{\prime}$

In the light of these results, the reduced voltage at any point $Q$ of the helix can be reconstructed via the OSI formula:

$\tilde{V}(\xi)=\sum_{n=n_{0}-q+1}^{n_{0}+q} \tilde{V}\left(\xi_{n}\right) D_{N}\left(\xi-\xi_{n}\right) \Omega_{N^{\prime \prime}}\left(\xi-\xi_{n}\right)$

where $n_{0}=\operatorname{Int}\left[\left(\xi-\xi\left(\phi_{i}\right)\right) / \Delta \xi\right]$ is the index of the sample nearest (on the left) to $Q, 2 q$ is the number of retained samples and

$\xi_{n}=\xi\left(\phi_{i}\right)+n \Delta \xi=\xi\left(\phi_{i}\right)+2 \pi n /(2 N+1)$

$N=\operatorname{Int}\left[\chi N^{\prime}\right]+1 ; \quad N^{\prime}=\operatorname{Int}\left[\chi^{\prime} W_{\xi}\right]+1$

Moreover,

$D_{N}(\xi)=\frac{\sin ((2 N+1) \xi / 2)}{(2 N+1) \sin (\xi / 2)}$

$\Omega_{N^{\prime \prime}}(\xi)=\frac{T_{N^{\prime \prime}}\left[2(\cos (\xi / 2) / \cos (\bar{\xi} / 2))^{2}-1\right]}{T_{N^{\prime \prime}}\left[2 / \cos ^{2}(\bar{\xi} / 2)-1\right]}$

are the Dirichlet and Chebyshev Sampling functions, respectively, $T_{N^{\prime \prime}}(\cdot)$ being the Chebyshev polynomial of degree $N^{\prime \prime}$ $=N-N^{\prime}$ and $\bar{\xi}=q \Delta \xi$.

The OSI formula (13) can be used to evaluate the "intermediate samples", namely, the reduced voltage values at the intersection points between the helix and the generatrix passing through $P$. Once these samples have been evaluated, the reduced voltage at $P$ can be reconstructed via the following OSI expansion:

$\tilde{V}(\vartheta, \varphi)=\sum_{m=m_{0}-p+1}^{m_{0}+p} \tilde{V}\left(\vartheta_{m}\right) D_{M}\left(\vartheta-\vartheta_{m}\right) \Omega_{M^{\prime}}\left(\vartheta-\vartheta_{m}\right)$

where $\vartheta_{m}=\vartheta_{m}(\varphi)=\vartheta\left(\phi_{i}\right)+k \varphi+m \Delta \vartheta=\vartheta_{0}+m \Delta \vartheta, m_{0}=$ $\operatorname{Int}\left[\left(\vartheta-\vartheta_{0}\right) / \Delta \vartheta\right], M^{\prime \prime}=M-M^{\prime}, \tilde{V}\left(\vartheta_{m}\right)$ are the intermediate samples, and the other symbols have the same meaning as in (13). It is so possible to reconstruct the NF data required to carry out the classical NF-FF transformation with cylindrical scanning [22].

\section{EXPERIMENTAL VALIDATION}

Some experimental results assessing the validity of the described NF-FF transformation technique with helicoidal scanning are reported in this section.

The laboratory tests have been carried out in the anechoic chamber available at the laboratory of antenna characterization of the University of Salerno, which is provided with a NF facility system supplied by MI Technologies. The chamber, whose dimensions are $8 \mathrm{~m} \times 5 \mathrm{~m} \times 4 \mathrm{~m}$, is provided with pyramidal absorbers ensuring a background noise due to the residual reflections lower than $-40 \mathrm{~dB}$. A vertical scanner and a rotating table allow one to acquire the NF data at any point on a cylindrical surface surrounding the AUT. The rotating table MI-6111B, mounted with its rotary axis parallel to the vertical scanner, ensures an angular precision of $\pm 0.05^{\circ}$, whereas the vertical scanner, whose height is 240 $\mathrm{cm}$, is characterized by a linear precision of $\pm 0.005 \mathrm{~cm}$. The controller MI-4190, connected to a host computer by means of a IEEE-488 interface, is used to control the positioners motion and is completed by the option MI-4193, so that it is able to simultaneously drive both the positioners. The amplitude and phase measurements are performed by means of a vectorial network analyzer Anritsu 37247C. This last is computer-controlled and is characterized by wide dynamic range, high sensitivity and linearity over the range from 40 $\mathrm{MHz}$ to $20 \mathrm{GHz}$. An open-ended MI-6970-WR90 rectangular waveguide, whose end is tapered for minimizing the diffraction effects, is used as probe. The chamber is equipped in its quiet zone with an additional rotating table, which allows one to perform direct far-field measurements in the case of electrically small antennas.

The considered AUT is a MI-12-8.2 standard gain horn with aperture $19.4 \mathrm{~cm} \times 14.4 \mathrm{~cm}$, located on the plane $x=0$ of the adopted reference system (Fig. 1) and operating at the frequency of $10 \mathrm{GHz}$. Such an AUT has been modelled as enclosed in a sphere having radius equal to $12.08 \mathrm{~cm}$. The probe output voltages have been collected on a helix lying on a cylinder having $d=43.4 \mathrm{~cm}$ and $2 h=240 \mathrm{~cm}$.

The amplitudes of the reconstructed probe voltage relevant to the generatrices at $\varphi=0^{\circ}$ and $\varphi=30^{\circ}$ are compared in Figs. (3 and 4) with those directly measured on the same generatrices, in order to assess the effectiveness of the described two-dimensional OSI algorithm. As can be seen, there is an excellent agreement between the reconstructed voltage (crosses) and the measured one (solid line), except for the peripheral zone wherein the error is caused both by the truncation of the scanning zone and the environmental reflections. It is worth noting that the reconstructed voltage exhibits a smoother behaviour with respect to the measured one, since the spatial harmonics relevant to the noise sources outside the AUT spatial bandwidth are cut away, due to the filtering properties of the interpolation functions. For com- 
pleteness, the comparison between the phase of the recovered voltage and the measured one on the generatrix at $\varphi=$ $0^{\circ}$ is shown in Fig. (5) in the range $[-20 \mathrm{~cm}, 120 \mathrm{~cm}]$. Note that all the reconstructions have been obtained by using $\chi=1.20, \chi^{\prime}=1.30$, and $p=q=7$.

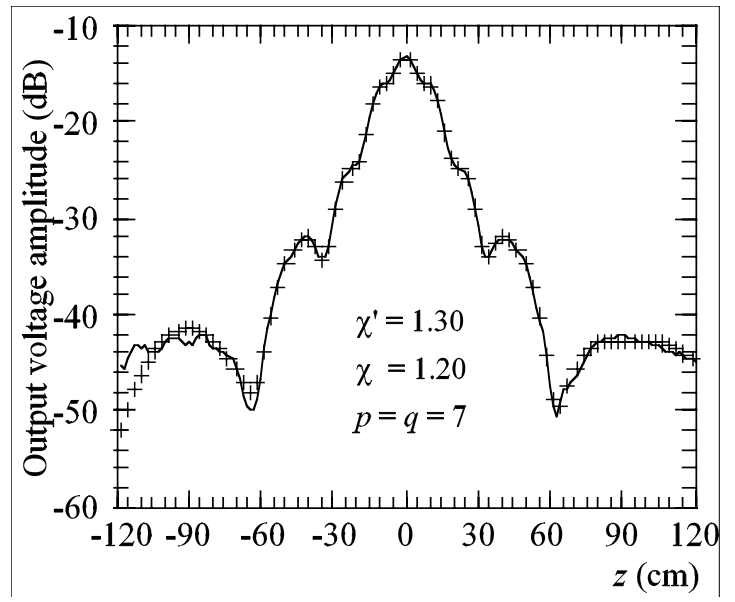

Fig. (3). Amplitude of the probe voltage on the generatrix at $\varphi=0^{\circ}$. Solid line: measured. Crosses: interpolated.

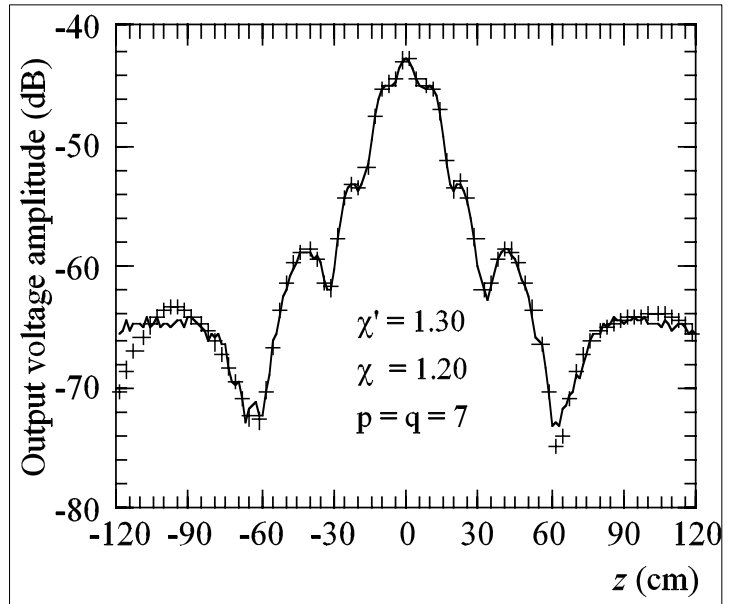

Fig. (4). Amplitude of the probe voltage on the generatrix at $\varphi=$ $30^{\circ}$. Solid line: measured. Crosses: interpolated.

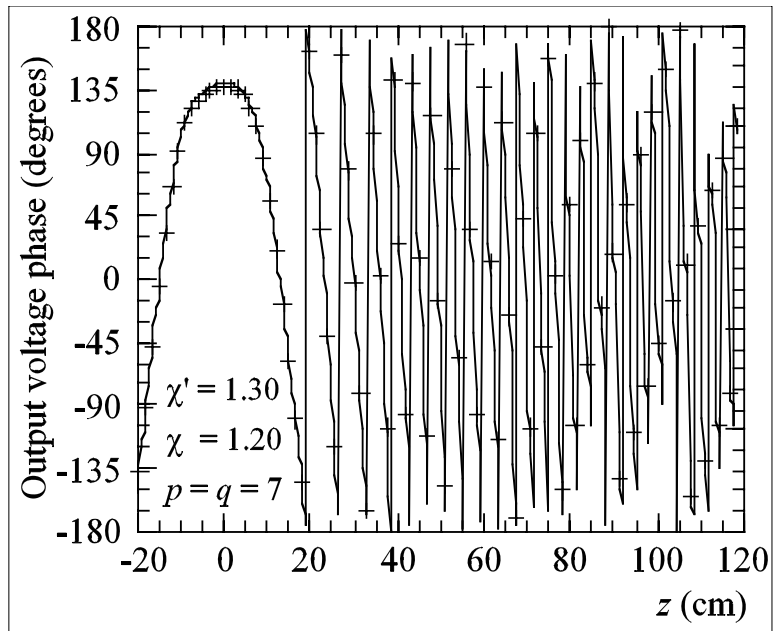

Fig. (5). Phase of the probe voltage on the generatrix at $\varphi=0^{\circ}$. Solid line: measured. Crosses: interpolated.
The overall effectiveness of the described NF-FF transformation technique is assessed by comparing the FF pattern in the principal planes reconstructed from the collected helicoidal NF data with that obtained from the data directly measured on the classical cylindrical grid. In both the cases, the software package MI-3000 has been used to get the FF reconstructions. Obviously, the two-dimensional OSI algorithm has been employed for recovering the cylindrical data needed to perform the NF-FF transformation from the acquired helicoidal ones. As can be seen (Figs. 6 and 7), the FF reconstructions are very accurate, thus confirming the effectiveness of the approach.

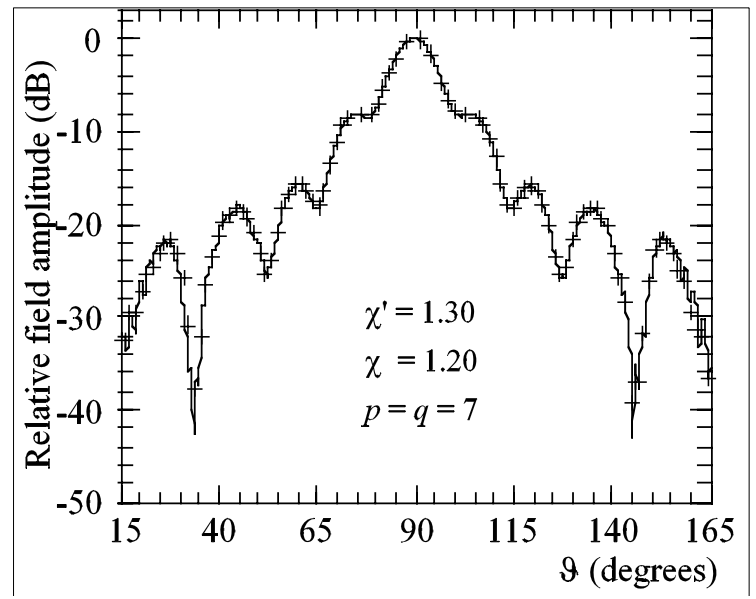

Fig. (6). E-plane pattern. Solid line: reference. Crosses: reconstructed from NF data acquired via helicoidal scanning.

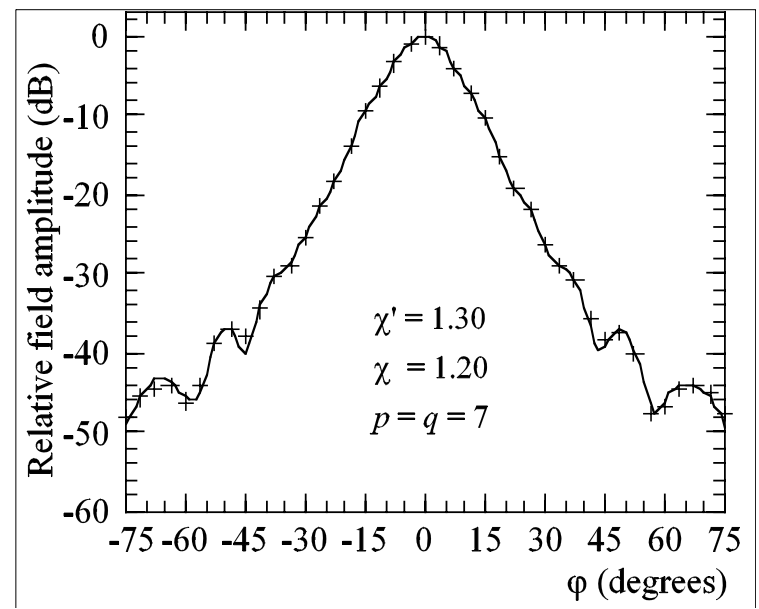

Fig. (7). H-plane pattern. Solid line: reference. Crosses: reconstructed from NF data acquired via helicoidal scanning.

It is worthy to note that the number of data needed by such a NF-FF transformation is 1922 , whereas that required by MI software package to cover the same scanning zone is 11520 . As shown, the helicoidal scanning allows one to remarkably reduce the number of measurements, without losing the accuracy of the classical approach. Note that the number of employed data is comparable with that (2118) needed by the nonredundant NF-FF transformation with cylindrical scan [8] and is significantly less than that needed by the NF helicoidal scanning technique [23], which requires 
the same number and retains the same accuracy of the classical approach.

At last, the E-plane and H-plane FF patterns obtained from the helicoidal measurements are compared in Figs. (8 and 9) with that directly measured in the FF zone. As can be seen, also in this case there is a very good agreement, thus further assessing the validity of the proposed NF-FF transformation with helicoidal scanning. The discrepancies in the reconstruction of the E-plane far-out sidelobes (see Fig. 8) are obviously due to the truncation of the NF scanning zone.

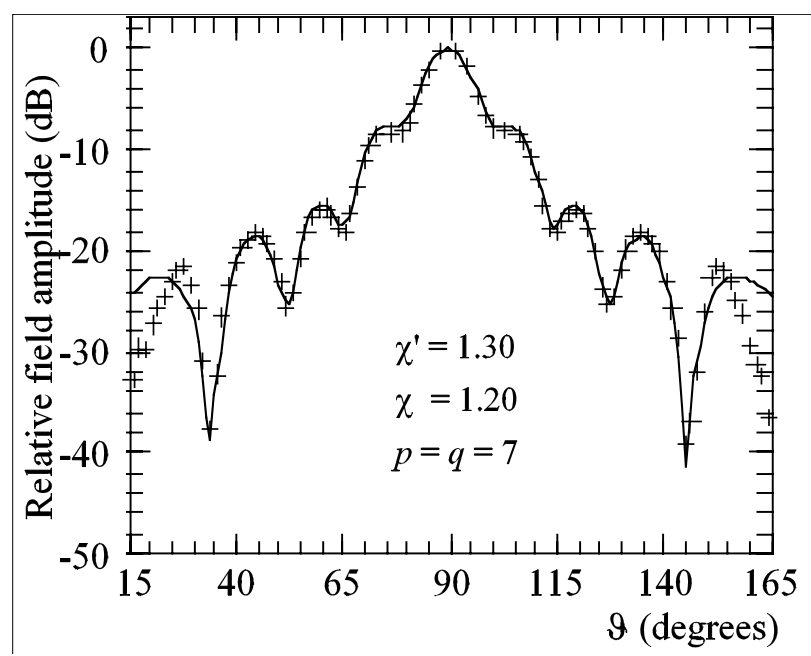

Fig. (8). E-plane pattern. Solid line: direct FF measurements. Crosses: reconstructed from NF data acquired via helicoidal scanning.

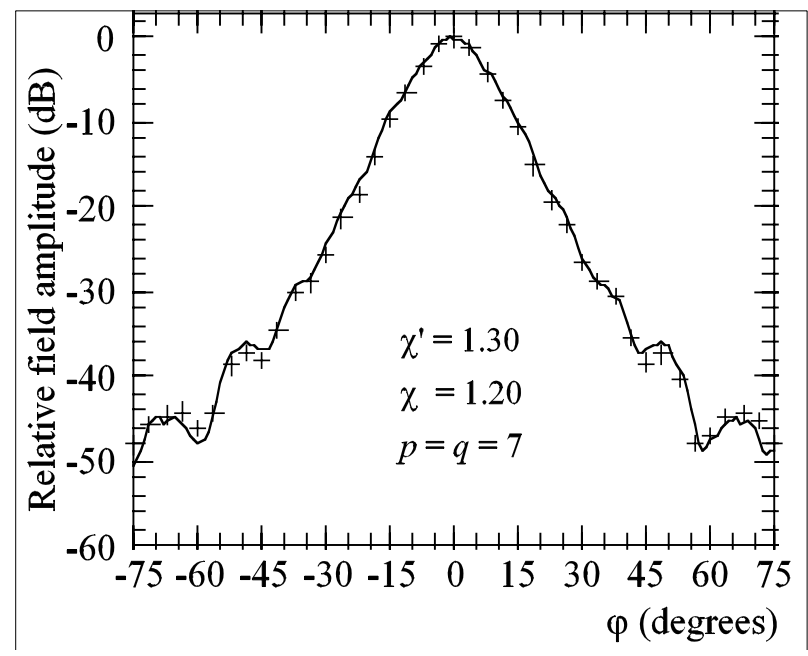

Fig. (9). H-plane pattern. Solid line: direct FF measurements. Crosses: reconstructed from NF data acquired via helicoidal scanning.

\section{REFERENCES}

[1] "Special Issue on near-field scanning techniques", IEEE Trans. Antennas Propag., vol. AP-36, pp. 727-901, June 1988.

[2] A.D. Yaghjian, "An overview of near-field antenna measurements", IEEE Trans. Antennas Propag., vol. AP-34, pp. 30-45, January 1986.
[3] C. Gennarelli, G. Riccio, F. D'Agostino, and F. Ferrara, "NearField - Far-Field Transformation Techniques", vol. 1, CUES, Salerno, Italy, November 2004.

[4] O.M. Bucci, C. Gennarelli, G. Riccio, and C. Savarese, "Nearfield-far-field transformation from nonredundant plane-polar data: effective modellings of the source", IEE Proc. Microwaves Antennas Propag., vol. 145, pp. 33-38, 1998.

[5] O.M. Bucci, F. D'Agostino, C. Gennarelli, G. Riccio, and C. Savarese, "NF-FF transformation with plane-polar scanning: ellipsoidal modelling of the antenna", Automatika, vol. 41, pp. 159-164, 2000.

[6] F. D'Agostino, C. Gennarelli, G. Riccio, and C. Savarese, "Data reduction in the NF-FF transformation with bi-polar scanning", Microw. Opt. Technol. Lett., vol. 36, pp. 32-36, January 2003.

[7] O.M. Bucci, C. Gennarelli, G. Riccio, and C. Savarese, "NF-FF transformation with cylindrical scanning: an effective technique for elongated antennas", IEE Proc. Microwaves Antennas Propag., vol. 145, pp. 369-374, October 1998.

[8] O.M. Bucci, C. Gennarelli, G. Riccio, C. Savarese, and V. Speranza, "Non redundant representation of the electromagnetic fields over a cylinder with application to the near-field-far-field transformation", Electromagnetics, vol. 16, pp. 273-290, May 1996.

[9] F. D'Agostino, F. Ferrara, C. Gennarelli, G. Riccio, and C.Savarese, "NF-FF transformation with cylindrical scanning from a minimum number of data", Microw. Opt. Technol. Lett., vol. 35, pp. 264-270, November 2002.

[10] O.M. Bucci, F. D'Agostino, C. Gennarelli, G. Riccio, and C. Savarese, "Data reduction in the NF-FF transformation technique with spherical scanning", J. Electromagn. Waves Appl., vol. 15, pp.755775, 2001.

[11] O.M. Bucci, C. Gennarelli, and C. Savarese, "Representation of electromagnetic fields over arbitrary surfaces by a finite and nonredundant number of samples", IEEE Trans. Antennas Propag., vol. 46, pp. 351-359, March 1998.

[12] O.M. Bucci, C. Gennarelli, and C. Savarese, "Fast and accurate near-field-far-field transformation by sampling interpolation of plane-polar measurements", IEEE Trans. Antennas Propag., vol. AP-39, pp. 48-55, January 1991.

[13] J.-C. Bolomey, B.J. Crown, G. Fine, L. Jofre, M. Mostafavi, D. Picard, J.P. Estrada, P.G. Friederich, F.L. Cain, "Rapid near-field antenna testing via array of modulated scattering probes", IEEE Trans. Antennas Propag., vol. 36, pp. 804-814, June 1988.

[14] R.G. Yaccarino, L.I. Williams, and Y. Rahmat-Samii, "Linear spiral sampling for the bipolar planar antenna measurement technique", IEEE Trans. Antennas Propag., vol. AP-44, pp. 1049-1051, July 1996.

[15] O.M. Bucci, C. Gennarelli, G. Riccio, and C. Savarese, "Nonredundant NF-FF transformation with helicoidal scanning", $J$ Electromagn. Waves Appl., vol. 15, pp. 1507-1519, 2001.

[16] F. D'Agostino, C. Gennarelli, G. Riccio, and C. Savarese, "Theoretical foundations of near-field-far-field transformations with spiral scannings", Prog. Electromagn. Res., vol. PIER 61, pp. 193214, 2006.

[17] O.M. Bucci, F. D’Agostino, C. Gennarelli, G. Riccio, and C. Savarese, "Probe compensated FF reconstruction by NF planar spiral scanning", IEE Proc. Microwaves Antennas Propag., vol. 149, pp. 119-123, April 2002.

[18] O.M. Bucci, F. D’Agostino, C. Gennarelli, G. Riccio, and C. Savarese, "NF-FF transformation with spherical spiral scanning", IEEE Antennas Wireless Propag. Lett., vol. 2, pp. 263-266, 2003.

[19] O.M. Bucci, G. D'Elia, and M.D. Migliore, "Advanced field interpolation from plane-polar samples: experimental verification", IEEE Trans. Antennas Propag., vol. 46, pp. 204-210, February 1998.

[20] O.M. Bucci and G. Franceschetti, "On the spatial bandwidth of scattered fields", IEEE Trans. Antennas Propag., vol. AP-35, pp. 1445-1455, December 1987. 
[21] C. Gennarelli, G. Riccio, V. Speranza, and C. Savarese, "Fast and accurate interpolation of radiated fields over a cylinder", Prog. Electromagn. Res., vol. PIER 8, pp. 349-375, 1994.

[22] W.M. Leach Jr. and D.T. Paris, "Probe compensated NF measurements on a cylinder", IEEE Trans. Antennas Propag., vol. AP-21, pp. 435-445, July 1973.
[23] S. Costanzo and G. Di Massa, "Far-field reconstruction from phaseless near-field data on a cylindrical helix", J. Electromagn. Waves Appl., vol. 18, pp. 1057-1071, 2004.

Received: September 08, 2009

Revised: November 10, 2009

Accepted: November 27, 2009

(c) D'Agostino et al.; Licensee Bentham Open.

This is an open access article licensed under the terms of the Creative Commons Attribution Non-Commercial License (http://creativecommons.org/licenses/by-nc/3.0/) which permits unrestricted, non-commercial use, distribution and reproduction in any medium, provided the work is properly cited. 